\title{
Bloch-Redfield-Wangsness theory engine implementation using symbolic processing software
}

\author{
Ilya Kuprov *, Nicola Wagner-Rundell, P.J. Hore \\ Department of Chemistry, University of Oxford, Physical and Theoretical Chemistry Laboratory, South Parks Road, Oxford OX1 3QZ, UK
}

Received 4 July 2006; revised 28 August 2006

Available online 3 November 2006

\begin{abstract}
We describe a general method for the automated symbolic processing of Bloch-Redfield-Wangsness relaxation theory equations for liquid-phase spin dynamics in the algebraically challenging case of rotationally modulated interactions. The processing typically takes no more than a few seconds (on a contemporary single-processor workstation) and yields relaxation rate expressions that are completely general with respect to the spectral density functions, relative orientations, and magnitudes of the interaction tensors, with all cross-correlations accounted for. The algorithm easily deals with fully rhombic interaction tensors, and is able, with little if any modification, to treat a large variety of the relaxation mechanisms encountered in NMR, EPR, and spin dynamics in general.
\end{abstract}

(C) 2006 Elsevier Inc. All rights reserved.

Keywords: Relaxation theory; Symbolic processing; Wigner function; Irreducible spherical tensor; Rotation group; Software

\section{Introduction}

The relaxation of a perturbed spin system to thermal equilibrium is ubiquitous in all forms of magnetic resonance spectroscopy [1]. In part responsible for the very existence of NMR and EPR signals, relaxation yields valuable structural information on inter-particle distances (from dipolar relaxation [1-3]), relative orientations and angles (from cross-correlated relaxation [4-6]), and order parameters and motional correlation times (from the magnetic field dependence of relaxation rates [7]).

Most practical techniques for the analysis of spin relaxation data rely on a compact and elegant general treatment known as the Bloch-Redfield-Wangsness (BRW) theory, which is based on second-order time-dependent perturbation theory [8-11]. Although it works very well in most cases of practical importance, the intermediate algebraic expressions can be bulky. As density matrix dimensions scale exponentially with the number of spins and each

\footnotetext{
${ }^{*}$ Corresponding author. Fax: +44 1865275410.

E-mail address: ilya.kuprov@chem.ox.ac.uk (I. Kuprov).
}

anisotropic interaction adds another set of directional functions, the formulae quickly become inconveniently cumbersome [12-14].

With the spectacular advances in symbolic processing software in recent years, in particular Mathematica [15], it is only a matter of time before the tedious inner workings of the analytical BRW theory are relegated to computers. This, however, is not a trivial task, and we share in this communication our experience in setting up such a system. The algorithm occupies only a page of code, but easily deals with the relaxation analysis of a large variety of systems-from simple dipole-dipole/chemical shift anisotropy cross-correlations to zero-field-splitting-induced relaxation of high-spin electron shells, and beyond, providing an extension to current programs [16,17], which have so far used hand-coded relaxation superoperators. In the approach described here, the underlying idea is to identify and supply the symbolic engine with the bare minimum of information to ensure successful processing, at the same time avoiding irrelevant attempts to simplify and transform intermediate results. Although we provide specific examples and (Mathematica-based [15]) implementations, we have chosen to focus here on the general strategy rather 
than on details of coding and usage, since the latter may vary from one programming environment to another.

\section{Equations of BRW theory}

The 'master equation' for the evolution of the density operator of BRW theory has the following form:

$$
\begin{aligned}
\frac{\partial \hat{\rho}(t)}{\partial t}= & -\mathrm{i}\left[\hat{H}_{0}, \hat{\rho}(t)-\hat{\rho}^{\mathrm{eq}}\right] \\
& -\sum_{n, m} \int_{0}^{\infty} g_{n m}(\tau)\left[\hat{K}_{n},\left[\mathrm{e}^{\mathrm{i} \hat{H}_{0} \tau} \hat{K}_{m}^{\dagger} \mathrm{e}^{-\mathrm{i} \hat{H}_{0} \tau}, \hat{\rho}(t)-\hat{\rho}^{\mathrm{eq}}\right]\right] \mathrm{d} \tau .
\end{aligned}
$$

(See the recent review by Goldman [9] and references therein for a detailed derivation and analysis of the required assumptions.) In this equation, the spin Hamiltonian is separated into the static part $\hat{H}_{0}$ and the dynamic part $\hat{H}_{1}(t)$, such that

$$
\hat{H}(t)=\hat{H}_{0}+\hat{H}_{1}(t), \quad \overline{\hat{H}_{1}(t)}=\hat{0}, \quad\left|\hat{H}_{1}(t)\right| \ll\left|\hat{H}_{0}\right|,
$$

where the overbar denotes an ensemble average (or a temporal average, if we accept the ergodic hypothesis). $\hat{K}_{j}$ are the static basis operators in an expansion of the timedependent part of the Hamiltonian

$\hat{H}_{1}(t)=\sum_{n} f_{n}(t) \hat{K}_{n}$

and $g_{n m}(\tau)$ are the correlation functions of the scalar stationary stochastic processes $f_{n}(t)$ in this expansion

$\overline{f_{n}(t) f_{m}^{*}(t+\tau)}=\overline{f_{n}(0) f_{m}^{*}(\tau)}=g_{n m}(\tau)$.

One can use $\hat{\rho}(t)-\hat{\rho}^{\mathrm{eq}}$ in the first commutator in Eq. (1) because $\hat{\rho}^{\text {eq }}$, the equilibrium density operator, commutes with $\hat{H}_{0}$.

Eq. (1) is a system of first order ordinary differential equations in time $t$ for the elements of the density matrix. After the integral over $\tau$ has been evaluated, the final density matrix evolution equations have the following form [10]

$$
\frac{\partial \rho_{i j}}{\partial t}=\sum_{k, l}\left(-\mathrm{i} H_{i j k l}+R_{i j k l}\right)\left(\rho_{k l}-\rho_{k l}^{\mathrm{eq}}\right)
$$

in which $\hat{\hat{H}}$ is the commutation superoperator corresponding to $\hat{H}_{0}$, and $\hat{\hat{R}}$ is referred to as the relaxation superoperator. The optional last step in the derivation is to translate this system of equations into expressions for the physical observables using a suitable complete orthogonal operator basis set. The choice of the basis is a matter of convenience, but there are a few that are particularly useful [18]. Taking the trace with the chosen operator basis gives a final system of equations for the evolution of the corresponding full system of observables $A_{i}$

$$
\frac{\mathrm{d}}{\mathrm{d} t}\left\langle A_{i}\right\rangle=\sum_{j} k_{i j}\left(\left\langle A_{j}\right\rangle-\left\langle A_{j}^{\mathrm{eq}}\right\rangle\right) .
$$

It is the matrix of coefficients $k_{i j}$ in which we are interested. The elements of this matrix have first-order unitary contributions, arising from the relatively simple first term on the right hand side of Eq. (1). The non-unitary second-order contributions, which describe the relaxation, are bulky and benefit from automated symbolic processing.

\section{Symbolic processing: strategy}

Known spin relaxation mechanisms may be loosely divided into those caused by the stochastic modulation of scalar interactions (e.g., Fermi contact, $J$-coupling and exchange) or scalar multipliers of anisotropic interactions (e.g., distance-modulated dipolar), and the more mathematically demanding case of stochastic rotational modulation of anisotropic interactions. Algebraic complexity is a distinctive feature of the latter case, on which we therefore chose to concentrate in this communication.

It might appear that, since the integral in Eq. (1) is a linear superoperator independent of the density matrix on which it acts, simply typing it into e.g., Mathematica and requesting evaluation would be sufficient. This is not the case, chiefly because the explicit expressions for the correlation functions in Eq. (4) are in general unknown. Furthermore, the integral in Eq. (1) is a one-sided superoperator-valued Fourier transform of those correlation functions. Even if, sacrificing generality, we choose to assume a particular symbolic expression for $g_{n m}(\tau)$, a brute-force symbolic Fourier transform would be unacceptably time-consuming.

We outline below what we believe to be the optimal strategy for the symbolic processing of the relaxation theory equations, combining both speed and applicability to a broad range of relaxation-inducing interactions. Steps 1 and 2 are concerned with the preparation of the Hamiltonian, which may vary from one spin system to another, and generally follow the irreducible spherical tensor formalism pioneered by Freed [19,20] and Sanctuary [2125]. Steps 3 and 4 then detail the automated BRW theory processing steps.

An approximation frequently made when formulating the relaxation superoperator is to neglect all elements $R_{i j k l}$ that correspond to non-zero energy differences $\omega_{i j}-\omega_{k l} \neq 0$, since these may be shown to have a small long-term effect on the evolution of the density matrix [10]. This "secular" approximation is rather dangerous: it may distort numerical derivatives (encountered, e.g., in minimization algorithms) and fail in systems with overlapping transitions. We found that the symbolic processing software is sufficiently powerful to skip this approximation and arrive at the general expressions, which are valid for any system.

\subsection{Step 1: Expressing the interaction Hamiltonian in irreducible spherical tensor notation}

A convenient general feature of the anisotropic parts of spin interactions is that virtually all of them transform 
according to the $l=2$ irreducible representation of the rotation group. Consequently, the rotational modulation of these interactions may be treated in a very uniform and general way using the irreducible spherical tensor operator formalism [19-25].

For any traceless symmetric bilinear spin interaction written in its eigenframe, the translation into the irreducible spherical tensor notation is given by

$$
\begin{aligned}
\hat{\vec{S}} \cdot \mathbf{A} \cdot \hat{\vec{L}} & =a \hat{S}_{\mathrm{X}} \hat{L}_{\mathrm{X}}+b \hat{S}_{\mathrm{Y}} \hat{L}_{\mathrm{Y}}+c \hat{S}_{\mathrm{Z}} \hat{L}_{\mathrm{Z}} \\
& =\frac{a-b}{2} \hat{T}_{2,-2}+\frac{a-b}{2} \hat{T}_{2,2}+\frac{2 c-(a+b)}{\sqrt{6}} \hat{T}_{2,0},
\end{aligned}
$$

where $\hat{T}_{2, m}$ are the second rank irreducible spherical tensor operators $[26,27]$. The coefficients on the right hand side are proportional to the rhombicity and axiality of the tensor in question. Eq. (7) refers to the bilinear case (e.g., dipolar or anisotropic hyperfine interactions). In the case of a linear interaction such as the Zeeman interaction, one of the operator vectors is replaced by the external field vector, and in the case of quadratic interactions (such as zero-field splitting or the nuclear quadrupolar interaction), the operator vectors on both sides of the interaction tensor refer to the same spin. For the sake of completeness we will also provide the reverse relations, which take the rank-2 irreducible spherical tensors back to the two-spin product operator basis:

$$
\begin{aligned}
& \hat{T}_{2,2}=+\frac{1}{2} \hat{S}_{+} \hat{L}_{+}, \\
& \hat{T}_{2,1}=-\frac{1}{2}\left(\hat{S}_{\mathrm{Z}} \hat{L}_{+}+\hat{S}_{+} \hat{L}_{\mathrm{Z}}\right), \\
& \hat{T}_{2,0}=+\sqrt{\frac{2}{3}}\left(\hat{S}_{\mathrm{Z}} \hat{L}_{\mathrm{Z}}-\frac{1}{4}\left(\hat{S}_{+} \hat{L}_{-}+\hat{S}_{-} \hat{L}_{+}\right)\right), \\
& \hat{T}_{2,-1}=+\frac{1}{2}\left(\hat{S}_{\mathrm{Z}} \hat{L}_{-}+\hat{S}_{-} \hat{L}_{\mathrm{Z}}\right), \\
& \hat{T}_{2,-2}=+\frac{1}{2} \hat{S}_{-} \hat{L}_{-} .
\end{aligned}
$$

Transformation (7) is the critical first step in preparation for automated symbolic processing because the irreducible spherical tensors have well-defined rotation properties and correlation functions [12,27]. This enables their manipulation and processing to be coded compactly later in the algorithm. Another important aspect is that the transformation in Eq. (7) only needs to be performed once - it has the same functional form for the majority of symmetric traceless interactions in spin dynamics. From system to system only the operator definitions (8) may need to change, simply because the spin quantum numbers and therefore the matrix dimensions may differ.

\subsection{Step 2: Setting up rotations}

After the eigenframe expressions for the interaction Hamiltonians have been obtained (with variable, pre-measured or pre-computed eigenvalues), the tensors need to be positioned in the molecular frame, and an overall molecular rotation has to be set up. The irreducible spherical tensors of a given rank transform into each other under any rotation [27] according to

$\hat{\hat{R}}(\alpha, \beta, \gamma) \hat{T}_{l, m}=\sum_{m^{\prime}=-l}^{l} \hat{T}_{l, m^{\prime}} \mathbf{D}_{m^{\prime}, m}^{(l)}(\alpha, \beta, \gamma)$.

In this expression $\hat{\hat{R}}(\alpha, \beta, \gamma)$ denotes a rotation with (possibly time-dependent) Euler angles $\alpha, \beta, \gamma$. The coefficients $\mathfrak{D}_{m^{\prime}, m}^{(l)}(\alpha, \beta, \gamma)$ are Wigner functions [26,27]. The three Euler angles position the interaction tensor relative to the molecular frame and describe any internal conformational motion that might affect that tensor. After the rotation is applied, Eq. (7) transforms into

$$
\begin{aligned}
\hat{\hat{R}}(\hat{\vec{S}} \cdot \mathbf{A} \cdot \hat{\vec{L}})= & \frac{a-b}{2} \sum_{m^{\prime}=-2}^{2} \hat{T}_{2, m^{\prime}} \mathfrak{D}_{m^{\prime},-2}^{(2)}+\frac{a-b}{2} \sum_{m^{\prime}=-2}^{2} \hat{T}_{2, m^{\prime}} \mathfrak{D}_{m^{\prime}, 2}^{(2)} \\
& +\frac{2 c-(a+b)}{\sqrt{6}} \sum_{m^{\prime}=-2}^{2} \hat{T}_{2, m^{\prime}} \mathfrak{D}_{m^{\prime}, 0}^{(2)} .
\end{aligned}
$$

For the purposes of BRW theory, the symbolic processor never needs explicit expressions for the Wigner functions because they are replaced when Eq. (4) is applied. The explicit definitions are sometimes useful at the very last stage of the symbolic processing of cross-correlated relaxation, where a few Wigner functions encoding the relative orientation of the interaction tensors survive and need to be translated into trigonometric functions [27].

For multiple rotations, Eq. (9) is applied several times sequentially. Each rotation generally has its own set of (possibly time-dependent) Euler angles. The overall molecular rotation is special in this respect: its Euler angles are shared by all the interaction tensors in the system. In the processing stage this automatically accounts for every cross-correlation present in the system. The cross-correlations are thus naturally accounted for without requiring special treatment.

Because the orientations of the interaction tensors in the molecular frame are usually treated as fixed, the associated rotational functions are simply constants. Eq. (10) then simplifies to

$$
\begin{aligned}
& \hat{\hat{R}}_{\mathrm{pos}}(\hat{\vec{S}} \cdot \mathbf{A} \cdot \hat{\vec{L}})=\sum_{m=-2}^{2} \hat{T}_{2, m} \Phi_{m}, \\
& \Phi_{m}=\frac{a-b}{2}\left(\mathfrak{D}_{m,-2}^{(2)}+\mathfrak{D}_{m, 2}^{(2)}\right)+\frac{2 c-(a+b)}{\sqrt{6}} \mathfrak{D}_{m, 0}^{(2)},
\end{aligned}
$$

where $\hat{\hat{R}}_{\text {pos }}$ denotes a 'positioning' rotation which takes the interaction from its eigenframe into the molecular frame; the quantities $\Phi_{m}$ are scalars which may be treated as constants throughout the calculation. Not having to consider them explicitly leads to a significant simplification at the processing stage: the only property of the $\Phi_{m}$ that needs to be declared is their behaviour under the complex conjugate operation 
$\Phi_{m}^{*}=(-1)^{m} \Phi_{-m}$

As we shall see below, Eqs. (7)-(12) allow any rotationally induced relaxation treatment to be performed in full generality with respect to the correlation functions/spectral densities and the principal values and orientations of the interaction tensors involved, including the case of anisotropic rotational diffusion.

\subsection{Step 3: Processing correlation functions using upvalues}

The ensemble averaging performed in BRW theory, together with the assumption that the molecular reorientation is a stationary stochastic process, leads to products of differently timed Wigner functions being replaced by a single function of their time separation $\tau$-the motional correlation functions $g(\tau)[9]$

$\overline{\mathfrak{M}_{a, b}^{(l)}(t) \mathfrak{M}_{c, d}^{(k)}(t+\tau)}=\overline{\mathfrak{M}_{a, b}^{(l)}(0) \mathfrak{M}_{c, d}^{(k)}(\tau)}=\frac{\delta_{l k}}{2 l+1} g_{a b c d}(\tau)$.

Here and below $\mathfrak{M}_{a, b}^{(l)}(t)$ denotes a Wigner function corresponding to the overall molecular rotation. The normalization on the right hand side of Eq. (13) is chosen to ensure that the correlation function, as the name suggests, equals unity at zero time separation. Except in very special cases (e.g., with a strongly anisotropic rotational diffusion tensor), most of the $g_{a b c d}(\tau)$ functions are zero and the overall rotational motion can be adequately described by a single correlation function.

Due to lack of concrete knowledge about $g_{a b c d}(\tau)$ in the ensemble in question, and also because Wigner functions are rather bulky, the direct analytical evaluation of the left hand side of Eq. (13) is not possible. Although Replace type directives of the symbolic processing kernel could be used to perform transformation (13), they fail in practice (they rely on strict pattern matching and are easily defeated by, for example, an unopened bracket or a minus sign). We shall therefore proceed to use the more powerful concept of upvalues [15], hereafter denoted by the $\hat{=}$ sign

$\mathfrak{M}_{a_{-}, b_{-}}^{\left(l_{-}\right)}(0) \mathfrak{M}_{c_{-}, d_{-}}^{(k-)^{*}}(\tau) \hat{=} \frac{\delta_{l k}}{2 l+1} g_{a b c d}(\tau)$.

This upvalue is an instruction to the symbolic processing kernel to do the following throughout the subsequent calculations: to keep an eye on the occurrence of Wigner function products that match the left hand side of Eq. (14), and on encountering a match to replace it with the right hand side of (13). The variables with trailing underscores may stand for anything and are simply carried over to the right hand side. Note the profound difference between this upvalue statement and the explicit Replace command: while the latter is only performed once, transformation (13) is performed every time a suitable match is encountered by the kernel. It is also important to have this rule set as an upvalue. If it were a plain assignment, it would have been associated with the outermost object-a multiplication operator-and checked every time that object is encountered [15]. This would result in a dramatic slow-down. The upvalue, on the contrary, means that the rule is associated with the second object from the top, the Wigner functions, resulting in optimal performance [15].

Similarly, if two rotational motions are known to be uncorrelated (e.g., when the interaction tensors belong to different molecules or radicals), the upvalues for the correlations may be explicitly set to zero

$\mathfrak{M}_{a_{-}, b_{-}}^{\left(l_{-}\right)^{*}}(0) \mathfrak{R}_{c_{-}, d_{-}}^{\left(k_{-}\right)}(\tau) \hat{=} 0$,

$\mathfrak{N}_{a_{-}, b_{-}}^{\left(l_{-}\right)^{*}}(0) \mathfrak{M}_{c_{-,} d_{-}}^{\left(k_{-}\right)}(\tau) \hat{=} 0$.

In summary, upvalues (14) and (15) encode those few properties of Wigner functions that the symbolic processing kernel has to be aware of in order to perform the correlation function processing efficiently. This requires less than a second in all the examples given below because the explicit trigonometric expressions for the Wigner functions are never used; instead the calculation is performed exploiting the power and simplicity of on-the-fly patternmatching, which arguably corresponds to what a human mind would have done if it had the required speed and patience.

\subsection{Step 4: Custom-building the BRW integrator}

Once the correlation functions have been processed, the products of Wigner functions corresponding to overall rotation are replaced by the correlation functions $g(\tau)$. A superoperator-valued Fourier transform of these correlation functions now remains. The integrand in the right hand side of Eq. (1) is very uniform in structure, being a combination of $\mathrm{e}^{\mathrm{i} \omega \tau} g(\tau)$ terms with different values or symbols for $\omega$. Once again, due to lack of knowledge of the functional form of $g(\tau)$, a brute-force symbolic integration fails. The problem can be mitigated to some extent by assuming a particular functional expression for the correlation function (e.g. Lipari-Szabo bi-exponentials [28]), but any analytical evaluation of hundreds of Fourier transforms is still very slow. A much improved solution at this stage is not to rely on explicit integration, but rather to create a dedicated integrator which is only aware of a specific set of properties and is therefore very fast.

The basic property that any symbolic integrator ought to have is linearity

$\Upsilon\left(a_{-}+b_{-}\right):=\Upsilon(a)+\Upsilon(b)$,

$\Upsilon\left(a \_b-\right):=a \Upsilon(b) \quad$ if $a \in \mathbb{C} \vee a \in \mathbb{P}$.

The symbol $\mathbb{C}$ denotes a set of complex numbers and $\mathbb{P}$ the user-defined set of interaction parameters which do not have an explicit numerical value during the calculation (e.g., a variable tensor axiality - see Section 4 on how to define such a set). A downvalue sign $(:=)$ is used instead of an upvalue because we want to associate the rule with the integration operator itself rather than any particular 
integrand [15]. We can then proceed to define the integration rules for the typical expressions encountered during the symbolic processing of Eq. (1)

$\Upsilon\left(\mathrm{e}^{\mathrm{i} \omega-\tau} g(\tau)\right):=J(\omega)$,

where $J(\omega)$ is a spectral density function. The fact that the only rules specified for the integrator are (16) and (17) makes it very efficient: the vast tables of other possible integration routes are not consulted. Once the symbolic integration is complete, a trivial simplification command is sometimes required as the last stage to bring the equations to a form that a human mind perceives as elegant.
Because the processing of correlation functions is done in full generality, the resulting relaxation rates also contain imaginary contributions, called dynamic frequency shifts (DFS), which originate from the fact that the imaginary part of the Fourier transform (17) is in general small, but non-zero [29]. Obtaining expressions for the DFS is even more difficult than for the relaxation rates, because the spectral density $J(\omega)$ can no longer be assumed to be an even function, and the signs of frequencies therefore need to be controlled at every stage in the calculation. As we shall see in the examples below, this is naturally achieved in automated symbolic processing.

\section{Implementation examples}

\subsection{Example 1. Relaxation due to bilinear interaction anisotropy}

The rotational modulation of an anisotropic pair-wise bilinear interaction (interelectron dipolar, electron-nuclear hyperfine, internuclear dipolar, etc.) is often the dominant spin relaxation mechanism in a wide variety of systems. The Hamiltonian typically comprises an isotropic Zeeman interaction and the bilinear interaction in question, which we explicitly split into the isotropic part (which is sometimes absent and of which we will keep only the secular term ${ }^{1}$ ), and the anisotropic part

$$
\hat{H}=\omega_{1} \hat{L}_{\mathrm{Z}}+\omega_{2} \hat{S}_{\mathrm{Z}}+a \hat{L}_{\mathrm{Z}} \hat{S}_{\mathrm{Z}}+\hat{\vec{L}} \cdot \mathbf{A} \cdot \hat{\vec{S}}
$$

Splitting the Hamiltonian into $\hat{H}_{0}$ (Zeeman and scalar coupling) and $\hat{H}_{1}$ (anisotropic coupling), according to definition (2), and converting $\hat{H}_{1}$ to irreducible spherical tensors using Eqs. (7)-(10) then yields:

$$
\begin{aligned}
& \hat{H}_{0}=\omega_{1} \hat{L}_{\mathrm{Z}}+\omega_{2} \hat{S}_{\mathrm{Z}}+a \hat{L}_{\mathrm{Z}} \hat{S}_{\mathrm{Z}}, \\
& \hat{H}_{1}(t)=\frac{R h}{2} \sum_{m^{\prime}=-2}^{2} \hat{T}_{2, m^{\prime}} \mathfrak{M}_{m^{\prime},-2}^{(2)}(t)+\frac{R h}{2} \sum_{m^{\prime}=-2}^{2} \hat{T}_{2, m^{\prime}} \mathfrak{M}_{m^{\prime}, 2}^{(2)}(t)+\frac{A x}{\sqrt{6}} \sum_{m^{\prime}=-2}^{2} \hat{T}_{2, m^{\prime}} \mathfrak{M}_{m^{\prime}, 0}^{(2)}(t), \\
& A x=2 A_{\mathrm{ZZ}}-\left(A_{\mathrm{XX}}+A_{\mathrm{YY}}\right) \quad R h=A_{\mathrm{XX}}-A_{\mathrm{YY}},
\end{aligned}
$$

where $\mathfrak{M}_{m, k}^{(l)}(t)$ are Wigner functions corresponding to the overall molecular rotation and $\hat{T}_{2, m}$ are second rank irreducible spherical tensors linking spins $\mathrm{L}$ and $\mathrm{S}$, as defined in Eq. (8).

Transforming the Hamiltonian into the form shown in Eq. (19) finishes the manual preparation stage; all the rest is handled by the symbolic processing software. We will now describe the essential steps of the implementation. The full programs are given in the Supplementary Information. All notation, unless elaborated upon, is standard Mathematica syntax described in the manual [15]. We chose to consider spin-1/2 particles here, but arbitrary spin particles may be treated by replacing the Pauli matrices below with those appropriate for different spin quantum numbers.

We start by setting up the Kronecker ( $a k a$ direct or outer) product, the commutation operation and the operator scalar product [18]:

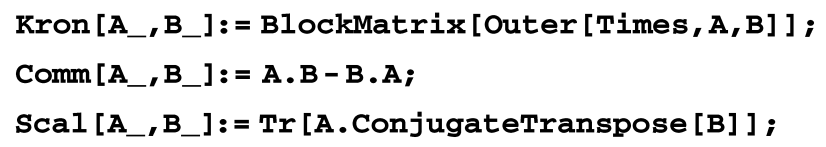

The basic single-spin operators, the identity operator, the first rank irreducible spherical tensors and the two-spin operators are then defined in a standard way $[16,17]$

\footnotetext{
${ }^{1}$ Non-secular terms of the isotropic coupling $a \hat{\vec{L}} \cdot \hat{\vec{S}}$ have been omitted from Eq. (18) for reasons of compactness: even though we can easily set up the full non-secular problem here, the resulting relaxation rate expressions would contain non-elementary functions (e.g., sums over roots of high-order polynomials) [30] and would not be readily comparable with literature results, which normally neglect the non-secular components of scalar couplings.
} 


$$
\begin{gathered}
I d=\left(\begin{array}{ll}
1 & 0 \\
0 & 1
\end{array}\right) ; \quad \sigma_{x}=\left(\begin{array}{cc}
0 & 1 / 2 \\
1 / 2 & 0
\end{array}\right) ; \quad \sigma_{y}=\left(\begin{array}{cc}
0 & -i / 2 \\
i / 2 & 0
\end{array}\right) ; \quad \sigma_{z}=\left(\begin{array}{cc}
1 / 2 & 0 \\
0 & -1 / 2
\end{array}\right) ; \\
T[1,-1]=\frac{\left(\sigma_{x}-i \sigma_{y}\right)}{\sqrt{2}} ; T[1,0]=\sigma_{z} ; T[1,1]=-\frac{\left(\sigma_{x}+i \sigma_{y}\right)}{\sqrt{2}} ; \\
L_{x}=\operatorname{Kron}\left[\sigma_{x}, I d\right] ; \quad L_{y}=\operatorname{Kron}\left[\sigma_{y}, I d\right] ; \quad L_{z}=\operatorname{Kron}\left[\sigma_{z}, I d\right] ; \quad L_{+}=L_{x}+i L_{y} ; \quad L_{-}=L_{x}-i L_{y} \\
S_{x}=\operatorname{Kron}\left[I d, \sigma_{x}\right] ; \quad S_{y}=\operatorname{Kron}\left[I d, \sigma_{y}\right] ; \quad S_{z}=\operatorname{Kron}\left[I d, \sigma_{z}\right] ; \quad S_{+}=S_{x}+i S_{y} ; S_{-}=S_{x}-i S_{y}
\end{gathered}
$$

To set up the second rank irreducible spherical tensors, it is convenient to use the direct product expression [26,27], which is more compact than the explicit definition via Eq. (8).

$$
T\left[2, q_{1}\right]:=(-1)^{\mathrm{q}} \sqrt{5} \sum_{\mathrm{q} 1=-1}^{1} \sum_{\mathrm{q}^{2}=-1}^{1}(\mathrm{ThreeJSymbol}[\{1, \mathrm{q} 1\},\{1, \mathrm{q} 2\},\{2,-\mathrm{q}\}] \operatorname{Kron}[\mathrm{T}[1, \mathrm{q} 1], \mathrm{T}[1, \mathrm{q} 2]]) \text {; }
$$

Since Mathematica assumes nothing about the variables of Hamiltonian (18), they have to be explicitly declared as being real. The separate InteractionParameters list is created for this purpose to avoid overloading the global Assumptions variable because of the associated performance impact. The membership of a particular variable in Parameters is then verified by checking if it appears on the InteractionParameters list:

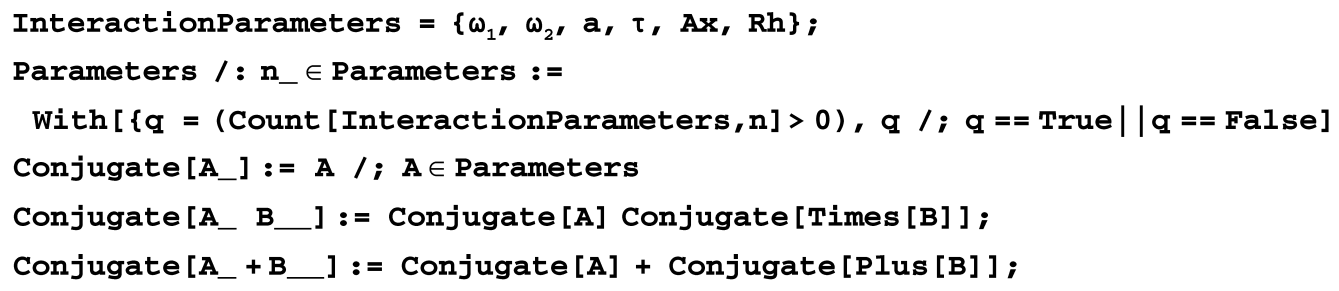

The third definition tells the kernel to assume all members of the InteractionParameters list to be real, and the last two definitions state that the conjugation operation commutes with addition and multiplication. The double underscores mean that the rule will be threaded over multiple sequential multiplications and additions.

We now proceed to implement the upvalues discussed in Step 3 and the BRW integrator discussed in Step 4 in the Strategy section. We assume in this example that the molecule undergoes isotropic rotational diffusion, which can described by a single correlation function. Upvalue (14) is then implemented as

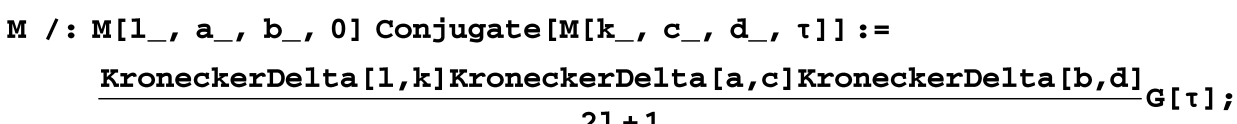

This tells the kernel to associate the pattern on the left hand side with the $\mathbb{M}$ symbol and, on encountering a match of the form $\mathfrak{M}_{a, b}^{(l)}(0) \mathfrak{M}_{c, d}^{(k)^{*}}(\tau)$, to replace it with $\frac{\delta_{l k} \delta_{a c} \delta_{b d}}{2 l+1} g(\tau)$. Downvalues (16), declaring BRW integrator linearity, are implemented as:

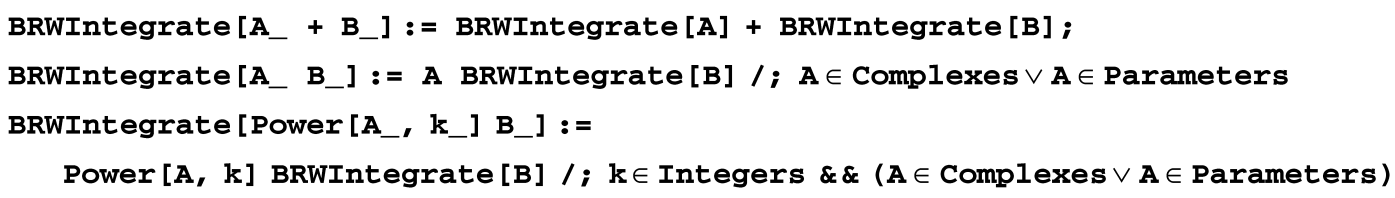

The powers of parameters have to be treated separately because the kernel is unaware of the fact that membership of Complexes and Parameters sets is inherited in the Power operation. The fastest and the most robust way to set up integration of a combination of $\mathrm{e}^{\mathrm{i} \omega \tau} g(\tau)$ terms with different numbers and symbols for $\omega$ appears to be:

BRWIntegrate $\left[e^{A_{-}}-[\tau]\right]:=J[S i m p l i f y[-i A / \tau]]$;

BRWIntegrate $[\mathrm{G}[\tau]]:=\mathrm{J}[0]$;

BRWIntegrate $[0]:=0$; 
where the first equation sets up downvalue (17) per se, and the other two deal with the special cases of unit exponential and zero argument, which do not fall, in the pattern matching sense, under the jurisdiction of the first equation.

We proceed by setting up the Hamiltonians as given in Eq. (19) and the double commutator from Eq. (1).

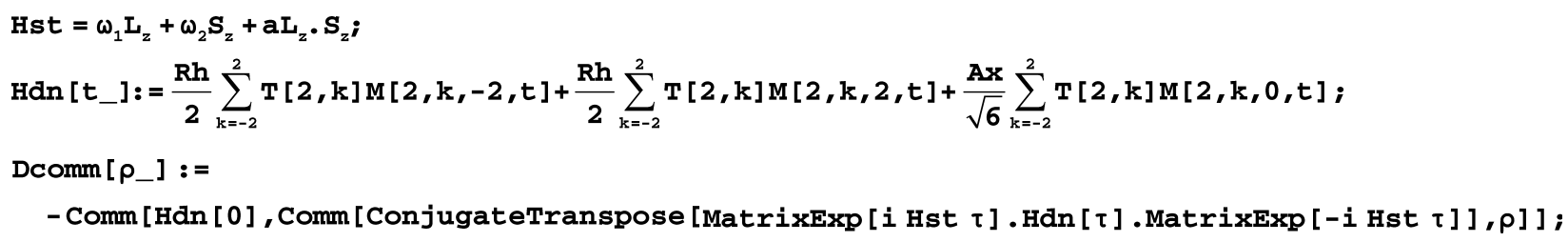

The rate of relaxation-induced transitions between the two observables corresponding to the two orthogonal operators $\hat{A}$ and $\hat{B}$ is then calculated as

$$
\text { Rate[A_, B_] := } \frac{\operatorname{Scal}[\mathrm{Dcomm}[\mathrm{A}], \mathrm{B}]}{\sqrt{\operatorname{Sca}[\text { [B, B] Scal [A, A] }}} / / \operatorname{Tr} \text { igToExp//ExpandA]1//BRWIntegrate//Simplify; }
$$

where we have used the fact that the integration and the matrix trace operation commute. This completes the implementation. Finally we present some illustrative answers:

$$
\begin{aligned}
& \text { Rate }\left[L_{\mathbf{z}}, \mathbf{L}_{\mathbf{z}}\right] \text { // Timing } \\
& \begin{aligned}
0.281 \text { Second, } & -\frac{A x^{2}+3 R h^{2}}{1440}\left(3 J\left[\frac{a}{2}-\omega_{1}\right]+3 J\left[-\frac{a}{2}-\omega_{1}\right]+3 J\left[-\frac{a}{2}+\omega_{1}\right]\right. \\
& \left.+3 J\left[\frac{a}{2}+\omega_{1}\right]+12 J\left[-\omega_{1}-\omega_{2}\right]+2 J\left[\omega_{1}-\omega_{2}\right]+2 J\left[-\omega_{1}+\omega_{2}\right]+12 J\left[\omega_{1}+\omega_{2}\right]\right)
\end{aligned} \\
& \text { Rate }\left[L_{z}, \mathbf{S}_{\mathbf{z}}\right] / / / \text { Timing } \\
& \qquad .219 \text { Second, } \frac{A x^{2}+3 R h^{2}}{720}\left(6 J\left[-\omega_{1}-\omega_{2}\right]-J\left[\omega_{1}-\omega_{2}\right]-J\left[-\omega_{1}+\omega_{2}\right]+6 J\left[\omega_{1}+\omega_{2}\right]\right) \\
& \text { Rate }\left[L_{+}, L_{+}\right] / / \text {Timing } \\
& 0.281 \text { Second, }-\frac{A x^{2}+3 R h^{2}}{1440}\left(8 J[0]+3 J\left[\frac{a}{2}-\omega_{1}\right]+3 J\left[-\frac{a}{2}-\omega_{1}\right]+3 J\left[\frac{a}{2}-\omega_{2}\right]\right. \\
& \left.+3 J\left[-\frac{a}{2}-\omega_{2}\right]+3 J\left[-\frac{a}{2}+\omega_{2}\right]+3 J\left[\frac{a}{2}+\omega_{2}\right]+12 J\left[-\omega_{1}-\omega_{2}\right]+2 J\left[-\omega_{1}+\omega_{2}\right]\right)
\end{aligned}
$$

Note that the often overlooked difference between $J(\omega)$ and $J(-\omega)$ is material because of the (usually small but non-zero) dynamic frequency shift component of the spectral density function, which is odd with respect to $\omega$. Obtaining the correct argument signs in the correlation function is crucially important in the ongoing hunt for the experimental observation of relaxation-induced dynamic frequency shifts [29]. If we now choose to neglect the DFS and put the scalar coupling constant $a$ to zero, we obtain the textbook rates for longitudinal relaxation, longitudinal cross-relaxation and transverse relaxation [31]:

$$
\begin{array}{ll}
\hat{L}_{\mathrm{Z}} \rightarrow \hat{L}_{\mathrm{Z}}: & -\frac{\mathrm{Ax}^{2}+3 R \mathrm{Rh}^{2}}{360}\left(3 \mathrm{~J}\left[\omega_{1}\right]+\mathrm{J}\left[\omega_{1}-\omega_{2}\right]+6 \mathrm{~J}\left[\omega_{1}+\omega_{2}\right]\right) \\
\hat{L}_{\mathrm{Z}} \rightarrow \hat{S}_{\mathrm{Z}}: & -\frac{A \mathrm{x}^{2}+3 R \mathrm{R}^{2}}{360}\left(\mathrm{~J}\left[\omega_{1}-\omega_{2}\right]-6 \mathrm{~J}\left[\omega_{1}+\omega_{2}\right]\right) \\
\hat{L}_{+} \rightarrow \hat{L}_{+}: & -\frac{A \mathrm{x}^{2}+3 R h^{2}}{720}\left(4 \mathrm{~J}[0]+3 \mathrm{~J}\left[\omega_{1}\right]+6 \mathrm{~J}\left[\omega_{2}\right]+\mathrm{J}\left[\omega_{1}-\omega_{2}\right]+6 \mathrm{~J}\left[\omega_{1}+\omega_{2}\right]\right)
\end{array}
$$

where in the case of isotropic rotational diffusion $J(\omega)=\tau_{c} /\left(1+\tau_{c}^{2} \omega^{2}\right)$, and the signs of the resulting rates conform to the definition of the relaxation superoperator given in Eq. (5).

\subsection{Example 2. Cross-correlation between two Zeeman interaction anisotropies}

The Hamiltonian of a two-spin system with anisotropic Zeeman interactions contains the constant term $\hat{H}_{0}$, corresponding to the isotropic part of either the $g$-factor or the chemical shielding, and the time-dependent term $\hat{H}_{1}(t)$ resulting from the rotational modulation of the anisotropies: 


$$
\begin{aligned}
& \hat{H}_{0}=\omega_{1} \hat{L}_{\mathrm{Z}}+\omega_{2} \hat{S}_{\mathrm{Z}} \\
& \hat{H}_{1}(t)=\hat{\hat{R}}_{\mathrm{mol}} \hat{\hat{R}}_{\mathrm{pos}}^{(1)}\left(\frac{R h_{1}}{2} \hat{T}_{2,-2}^{(1)}+\frac{R h_{1}}{2} \hat{T}_{2,2}^{(1)}+\frac{A x_{1}}{\sqrt{6}} \hat{T}_{2,0}^{(1)}\right)+\hat{\hat{R}}_{\mathrm{mol}} \hat{\hat{R}}_{\mathrm{pos}}^{(2)}\left(\frac{R h_{2}}{2} \hat{T}_{2,-2}^{(2)}+\frac{R h_{2}}{2} \hat{T}_{2,2}^{(2)}+\frac{A x_{2}}{\sqrt{6}} \hat{T}_{2,0}^{(2)}\right)
\end{aligned}
$$

where the two sets of axiality and rhombicity parameters refer to the two Zeeman tensors. $\hat{\hat{R}}_{\text {pos }}$ are 'positioning' rotations that take the Zeeman tensors from their eigenframes to the molecular frame, and $\hat{\hat{R}}_{\mathrm{mol}}$ is a global rotation of what is assumed to be a rigid molecule. We will not make any simplifying assumptions about the Zeeman tensors and consider the full rhombic case. After applying Eq. (11) the anisotropic part transforms into:

$$
\hat{H}_{1}(t)=\sum_{k, m=-2}^{2} \mathfrak{M}_{k, m}^{(2)}(t)\left[\hat{T}_{2, k}^{(\mathrm{L})} \Phi_{m}^{(\mathrm{L})}+\hat{T}_{2, k}^{(\mathrm{S})} \Phi_{m}^{(\mathrm{S})}\right]
$$

in which $\mathfrak{M}_{k, m}^{(2)}(t)$ are time-dependent Wigner functions corresponding to overall molecular rotation, $\Phi_{m}$ are defined in Eq. (11) and $\hat{T}_{2, k}$ are the irreducible spherical tensor operators, linking the spin operators and the external magnetic field.

A few more upvalues and assignments have to be made in addition to the ones specified in Example 1. Firstly, the kernel has to be made aware of Eq. (12):

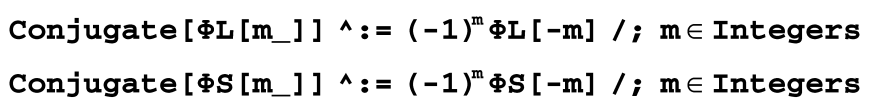

Secondly, because we are now dealing with Zeeman interactions, the irreducible spherical tensors (8) will couple spin projection operators to the external magnetic field. Because the magnetic field is assumed to be directed along the $Z$-axis of the laboratory frame (that is, $B_{\mathrm{X}}, B_{\mathrm{Y}}$, and $B_{ \pm}$are all zero), most of the terms in the definition (8) vanish:

$$
\begin{array}{llll}
\mathrm{T}[2,-2]=0 ; & \mathrm{T}[2,-1]=\frac{1}{2} \mathrm{~L}_{-} ; & \mathrm{T}[2,0]=\sqrt{\frac{2}{3} L_{z} ;} \quad \mathrm{T}[2,1]=-\frac{1}{2} L_{+} ; & \mathrm{T}[2,2]=0 ; \\
\mathrm{K}[2,-2]=0 ; & \mathrm{K}[2,-1]=\frac{1}{2} \mathrm{~S}_{-} ; & \mathrm{K}[2,0]=\sqrt{\frac{2}{3} S_{z} ;} \quad \mathrm{K}[2,1]=-\frac{1}{2} S_{+} ; \quad \mathrm{K}[2,2]=0 ;
\end{array}
$$

Defining the Hamiltonians completes the setup:

$$
\begin{aligned}
& \text { Hst }=\omega_{1} L_{z}+\omega_{2} S_{z} ; \\
& \text { Hdn }\left[t_{-}\right]:=\sum_{k=-2}^{2} \sum_{m=-2}^{2}(M[2, k, m, t](T[2, k] \Phi L[m]+K[2, k] \Phi S[m])) ;
\end{aligned}
$$

The Zeeman-Zeeman cross-correlation is known to affect only zero- and double-quantum coherences [32,33]. For the longitudinal magnetization and single-quantum coherence we have the usual independent rates [31]:

$$
\begin{aligned}
& \text { Rate }\left[L_{z}, L_{z}\right] / / \text { Timing } \\
& \quad 0.078 \text { Second, }-\frac{1}{10}\left(J\left[-\omega_{1}\right]+J\left[\omega_{1}\right]\right)\left(\Phi L[0]^{2}-2 \Phi L[-1] \Phi L[1]+2 \Phi L[-2] \Phi L[2]\right) \\
& \text { Rate }\left[L_{+}, L_{+}\right] / / \text {Timing } \\
& \quad 0.047 \text { Second, }-\frac{1}{30}\left(4 J[0]+3 J\left[-\omega_{1}\right]\right)\left(\Phi L[0]^{2}-2 \Phi L[-1] \Phi L[1]+2 \Phi L[-2] \Phi L[2]\right)
\end{aligned}
$$

Note the impressive timings. In these results the parameters and orientations of the Zeeman tensors in the molecular frame appear in their most general form, expressed via functions defined in Eq. (11). In this particular case we can use definitions (10) and explicit expressions for the Wigner functions [26,27] to simplify them further

$$
\Phi_{0}^{2}-2 \Phi_{-1} \Phi_{1}+2 \Phi_{-2} \Phi_{2}=\frac{A x^{2}+3 R h^{2}}{6}
$$

where the axiality and rhombicity now refer to the shielding tensor of spin $L$ :

$$
\begin{aligned}
& A x=\left[2 \sigma_{z z}-\left(\sigma_{x x}+\sigma_{y y}\right)\right] B_{0} \gamma, \\
& R h=\left(\sigma_{x x}-\sigma_{y y}\right) B_{0} \gamma .
\end{aligned}
$$

Just as in the Example 1, the spectral density function normalization is chosen so that it is a Fourier transform of a correlation function which equals one at zero time separation. This would yield $J(\omega)=\tau_{c} /\left(1+\tau_{c}^{2} \omega^{2}\right)+\mathrm{i} \omega \tau_{c}^{2} /\left(1+\tau_{c}^{2} \omega^{2}\right)$ in the isotropic tumbling approximation, which differs by a factor of $2 / 5$ from the definition given in Palmer's treatment [31]. The factor of 2/5 arises from the Wigner function normalization in Eq. (13) and from the fact that the Fourier transform in Eq. (1) is one-sided. It clearly does not belong to the spectral density function, so we decided to keep it elsewhere. 
In the case of zero-quantum coherence, where the cross-correlated relaxation is manifested, the expression for the relaxation rate is substantially more complex:

$$
\text { Rate } \begin{aligned}
\frac{\mathbf{1}}{\sqrt{\mathbf{2}}}\left(\mathbf{2} \mathbf{L}_{\mathbf{x}} \cdot \mathbf{S}_{\mathbf{x}}+\right. & \left.\left.\mathbf{2} \mathbf{L}_{\mathrm{y}} \cdot \mathbf{S}_{\mathrm{y}}\right), \frac{\mathbf{1}}{\sqrt{\mathbf{2}}}\left(\mathbf{2} \mathbf{L}_{\mathbf{x}} \cdot \mathbf{S}_{\mathbf{x}}+\mathbf{2} \mathbf{L}_{\mathbf{y}} \cdot \mathbf{S}_{\mathbf{y}}\right)\right] / / \text { Timing } \\
0.219 \text { Second, } & -\frac{2}{15} \mathrm{~J}[0]\left((\Phi L[0]-\Phi S[0])^{2}-2((\Phi L[-1]-\Phi S[-1])\right. \\
& \times(\Phi L[1]-\Phi S[1])-(\Phi L[-2]-\Phi S[-2])(\Phi L[2]-\Phi S[2]))) \\
& -\frac{1}{20}\left(J\left[-\omega_{1}\right]+\mathrm{J}\left[\omega_{1}\right]\right)\left(\Phi L[0]^{2}-2 \Phi L[-1] \Phi L[1]+2 \Phi L[-2] \Phi L[2]\right) \\
& -\frac{1}{20}\left(J\left[-\omega_{2}\right]+J\left[\omega_{2}\right]\right)\left(\Phi S[0]^{2}-2 \Phi S[-1] \Phi S[1]+2 \Phi S[-2] \Phi S[2]\right) .
\end{aligned}
$$

In practical calculations, the form above is the most convenient, because the expression for the directional functions $\Phi_{m}$ is compact (see Eqs. (11) and (22)). It is also the most general form for the fully rhombic case. After performing some cosmetic simplification, we have:

$$
\begin{aligned}
Z Q_{x} \rightarrow & Z Q_{x}:-\frac{1}{20} \frac{A x_{1}^{2}+3 R h_{1}^{2}}{6}\left[J\left(\omega_{1}\right)+J\left(-\omega_{1}\right)\right]-\frac{1}{20} \frac{A x_{2}^{2}+3 R h_{2}^{2}}{6}\left[J\left(\omega_{2}\right)+J\left(-\omega_{2}\right)\right] \\
& -\frac{2}{15} J(0)\left[\left(\Phi_{0}^{(\mathrm{L})}-\Phi_{0}^{(\mathrm{S})}\right)^{2}-2\left(\Phi_{-1}^{(\mathrm{L})}-\Phi_{-1}^{(\mathrm{S})}\right)\left(\Phi_{1}^{(\mathrm{L})}-\Phi_{1}^{(\mathrm{S})}\right)+2\left(\Phi_{-2}^{(\mathrm{L})}-\Phi_{-2}^{(\mathrm{S})}\right)\left(\Phi_{2}^{(\mathrm{L})}-\Phi_{2}^{(\mathrm{S})}\right)\right] .
\end{aligned}
$$

If we choose to expand it down to the bare trigonometric functions, it becomes:

$$
\begin{aligned}
& -\frac{1}{60}\left(\frac{A x_{1}^{2}+3 R h_{1}^{2}}{2}\left(J\left(-\omega_{1}\right)+J\left(\omega_{1}\right)\right)+\frac{A x_{2}^{2}+3 R h_{2}^{2}}{2}\left(J\left(-\omega_{2}\right)+J\left(\omega_{2}\right)\right)+R_{0} J(0)\right) \\
& R_{0}=\left(A x_{1} A x_{2} R h_{1} R h_{2}\right)\left(\begin{array}{cccc}
4 / 3 & A & 0 & B \\
A & 4 / 3 & C & 0 \\
0 & C & 4 & D \\
B & 0 & D & 4
\end{array}\right)\left(\begin{array}{l}
A x_{1} \\
A x_{2} \\
R h_{1} \\
R h_{2}
\end{array}\right) ; \\
& A=-\frac{1}{3}(1+3 \cos [2 \beta]) ; \quad B=-2 \cos [2 \gamma] \sin ^{2} \beta ; \quad C=-2 \cos [2 \alpha] \sin ^{2} \beta ; \\
& D=4 \sin [2 \alpha] \cos [\beta] \sin [2 \gamma]-\cos [2 \alpha](3+\cos [2 \beta]) \sin [2 \gamma]
\end{aligned}
$$

in which the three Euler angles $\alpha, \beta, \gamma$ define the relative orientation of the two Zeeman interaction tensors. The full expression (24) has not, to our knowledge, been reported in the literature, but its special case (NMR, Lipari-Szabo spectral densities, zero rhombicities, DFS neglected), published by Konrat and Sterk [32] and by Pellecchia et al. [33], is in complete accordance with the corresponding limit of Eq. (24).

Although it is fairly common to neglect shielding tensor rhombicities (in part because the rhombic expressions used to be difficult to derive), $a b$ initio calculations and experimental measurements indicate that shielding tensors of $\pi$-bonded carbons and nitrogens can be highly rhombic [34-36], with the difference between $\sigma_{\mathrm{XX}}$ and $\sigma_{\mathrm{YY}}$ often in excess of $30 \%$ of $\sigma_{\text {iso. }}$. For the relaxation rate of the $\mathrm{N}_{(\mathrm{H})}-\mathrm{C}_{(\mathrm{O})}$ zero-quantum coherence in a model Gly-Gly dipeptide (shielding tensors estimated from a B3LYP/6-31G(d,p)//CSGT B3LYP/cc-pV5Z calculation in explicit + PCM water using the Gaussian03 program [37], see Fig. 1), the neglect of rhombicity in Eq. (24) would lead to the underestimation of the resulting relaxation rate by $30-90 \%$, depending on the magnetic field and the rotational correlation time. Clearly, errors of this magnitude are unacceptable and the fully rhombic expressions for the relaxation rates should be preferred.

\subsection{Example 3. ZFS-induced relaxation in a spin-3/2 system}

The problem of spin relaxation induced by transient zero-field splitting (ZFS) arises, for example, in the description of electron spin dynamics in endofullerenes [38-40]. The spin Hamiltonian of the $\mathrm{N} @ \mathrm{C}_{60}$ endofullerene (ground electron term ${ }^{4} \mathrm{~S}$, i.e., spin $3 / 2$, with hyperfine coupling to ${ }^{14} \mathrm{~N}$ ) in a magnetic field has the following form:

$$
\hat{H}=\omega_{1} \hat{S}_{\mathrm{Z}}+\omega_{2} \hat{L}_{\mathrm{Z}}+a \hat{S}_{\mathrm{Z}} \hat{L}_{\mathrm{Z}}+\hat{\vec{S}} \cdot \mathbf{Z} \cdot \hat{\vec{S}}
$$

where $\omega_{1,2}$ are the Zeeman frequencies, $a$ is a hyperfine coupling constant (non-secular terms neglected) and $\mathbf{Z}$ is the ZFS tensor. The mathematical details of its rotation are identically the same as those for dipolar and CSA tensors, except we now have the same spin on both sides of the interaction matrix. After splitting up the Hamiltonian into the static and the dynamic part, we obtain: 


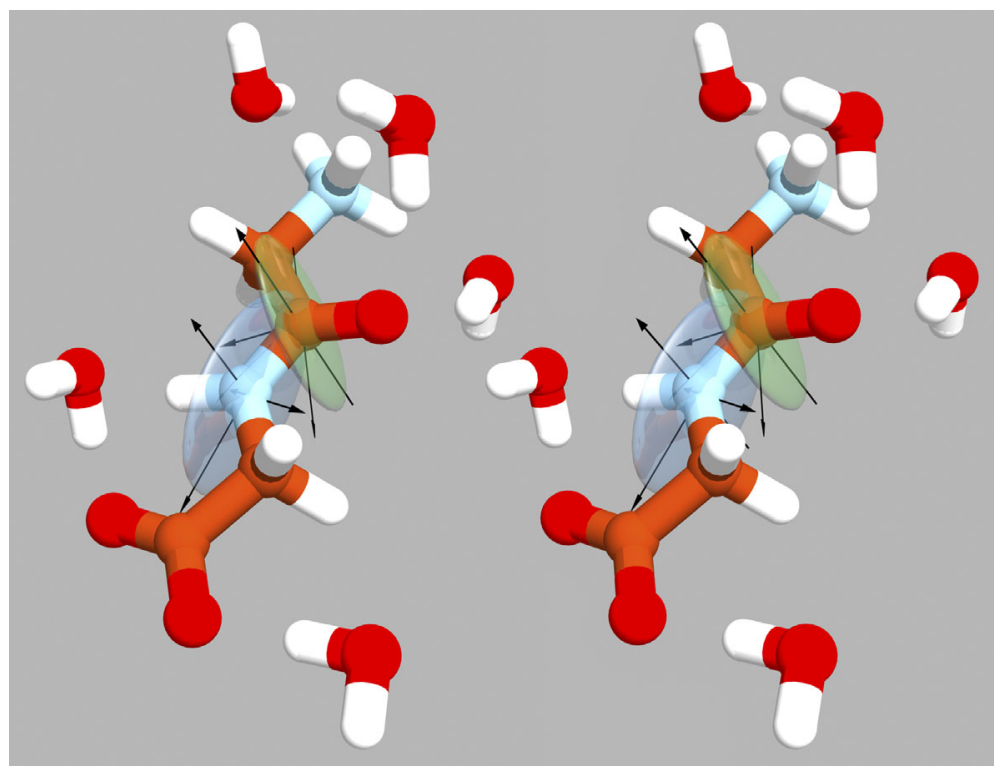

Fig. 1. Schematic stereo plot of the calculated absolute $\mathrm{N}_{(\mathrm{H})}$ and $\mathrm{C}_{(\mathrm{O})}$ shielding tensors in a Gly-Gly dipeptide. The Gaussian03 [37] log containing complete calculation details and shielding tensor data is included in the Supplementary Information.

$$
\begin{aligned}
& \hat{H}_{0}=\omega_{1} \hat{S}_{Z}+\omega_{2} \hat{L}_{\mathrm{Z}}+a \hat{S}_{\mathrm{Z}} \hat{L}_{\mathrm{Z}}, \\
& \hat{H}_{1}(t)=\frac{R h}{2} \sum_{m^{\prime}=-2}^{2} \hat{T}_{2, m^{\prime}} \mathfrak{M}_{m^{\prime},-2}^{(2)}(t)+\frac{R h}{2} \sum_{m^{\prime}=-2}^{2} \hat{T}_{2, m^{\prime}} \mathfrak{M}_{m^{\prime}, 2}^{(2)}(t)+\frac{A x}{\sqrt{6}} \sum_{m^{\prime}=-2}^{2} \hat{T}_{2, m^{\prime}} \mathfrak{M}_{m^{\prime}, 0}^{(2)}(t),
\end{aligned}
$$

where, just as in the case of Eq. (19), $\mathfrak{M}_{m, k}^{(l)}(t)$ are Wigner functions corresponding to the overall molecular rotation and $\hat{T}_{2, m}$ are second rank irreducible spherical tensors linking spin $S$ to itself, as defined in Eq. (8). The $A x$ and $R h$ parameters define the axiality and rhombicity of the ZFS tensor in the same way as in Eq. (19). Nothing changes in the program compared to Example 1, except the definitions of the Pauli matrices (now spin-3/2 and spin-1) and the fact that the definitions in Eq. (8) now have the same spin on both sides. After evaluation we get the following general expressions for the longitudinal and transverse electron relaxation rates:

\section{Rate $\left[S_{z}, S_{z}\right] / /$ Timing}

$$
\begin{aligned}
0.328 \text { Second, } & -\frac{A x^{2}+3 R h^{2}}{75}\left(J\left[-a-\omega_{1}\right]+J\left[a-\omega_{1}\right]+4 J\left[2 a-2 \omega_{1}\right]+4 J\left[-2 \omega_{1}\right]+J\left[-\omega_{1}\right]+J\left[\omega_{1}\right]\right. \\
& \left.+4 J\left[2 \omega_{1}\right]+J\left[-a+\omega_{1}\right]+4 J\left[-2 a-2 \omega_{1}\right]+J\left[a+\omega_{1}\right]+4 J\left[2 a+2 \omega_{1}\right]+4 J\left[-2 a+2 \omega_{1}\right]\right),
\end{aligned}
$$

Rate $\left[S_{+}, S_{+}\right] / /$Timing

$$
\begin{aligned}
0.297 \text { Second, } & -\frac{A x^{2}+3 R h^{2}}{75}\left(9 J[0]+3 J\left[-a-\omega_{1}\right]+3 J\left[a-\omega_{1}\right]+2 J\left[2 a-2 \omega_{1}\right]+2 J\left[-2 \omega_{1}\right]\right. \\
& \left.+3 J\left[-\omega_{1}\right]+2 J\left[\omega_{1}\right]+2 J\left[-a+\omega_{1}\right]+2 J\left[-2 a-2 \omega_{1}\right]+2 J\left[a+\omega_{1}\right]\right)
\end{aligned}
$$

which, after setting the scalar coupling parameter $a$ to zero and neglecting the dynamic frequency shifts, simplify to results reported by Knapp and co-workers [38,39]:

$$
\begin{aligned}
& \hat{S}_{\mathrm{Z}} \rightarrow \hat{S}_{\mathrm{Z}}:-\frac{2}{25}\left(A \mathrm{Ax}^{2}+3 \mathrm{Rh} \mathrm{h}^{2}\right)\left(\mathrm{J}\left[\omega_{1}\right]+4 \mathrm{~J}\left[2 \omega_{1}\right]\right) . \\
& \hat{S}_{+} \rightarrow \hat{S}_{+}: \quad-\frac{1}{25}\left(A x^{2}+3 R h^{2}\right)\left(3 \mathrm{~J}[0]+5 \mathrm{~J}\left[\omega_{1}\right]+2 \mathrm{~J}\left[2 \omega_{1}\right]\right) .
\end{aligned}
$$

The relaxation rates computed along the individual transitions were also found to be in complete agreement with those reported by Knapp et al. [38,39]. On a contemporary single-processor workstation, the full $144 \times 144$ symbolic relaxation superoperator for this system was computed in just under 30 seconds. 


\section{Conclusions}

We have described here what we believe to be a very general approach to the symbolic processing of Bloch-Redfield-Wangsness relaxation theory equations as applied to the liquid-phase spin dynamics in the mathematically demanding case of rotationally modulated interactions. The processing typically takes seconds (on a contemporary single-processor workstation) and yields relaxation rate expressions which are completely general with respect to the spectral density functions and relative orientations and magnitudes of the interaction tensors, with all crosscorrelations accounted for. The algorithm easily deals with fully rhombic cases, and is able, with little if any modification, to treat a variety of relaxation mechanisms.

\section{Acknowledgments}

We thank Mr. Christopher Rodgers, Dr. Arzhang Ardavan, and Dr. John Morton for enlightening discussions. I.K. is a Fellow by Examination at Magdalen College, Oxford. We thank the Oxford Supercomputing Centre for the generous allocation of CPU time.

\section{Appendix A. Supplementary data}

Supplementary data associated with this article can be found, in the online version, at doi:10.1016/j.jmr.2006. 09.023 .

\section{References}

[1] J. Kowalewski, L. Mäler, Nuclear Spin Relaxation in Liquids: Theory, Experiments, and Applications, Taylor \& Francis, London, 2006.

[2] A. Mittermaier, L.E. Kay, Science 312 (2006) 224-228.

[3] L. Banci, I. Bertini, C. Luchinat, Nuclear and Electron Relaxation: the Magnetic Nucleus-Unpaired Electron Coupling in Solution, VCH, Weinheim, Cambridge, 1991.

[4] C. Griesinger, Method Princ. Med. Chem. 16 (2003) 147-178.

[5] H. Schwalbe, T. Carlomagno, M. Hennig, J. Junker, B. Reif, C. Richter, C. Griesinger, Methods Enzymol. 338 (2001) 35-81.

[6] T. Carlomagno, C. Griesinger, J. Magn. Reson. 144 (2000) 280-287.

[7] A.G. Palmer III, Curr. Opin. Struct. Biol. 7 (1997) 732-737.

[8] A. Abragam, The Principles of Nuclear Magnetism, Clarendon Press, Oxford, 1961.

[9] M. Goldman, J. Magn. Reson. 149 (2001) 160-187.

[10] A.G. Redfield, in: J.S. Waugh (Ed.), Adv. Magn. Reson., Academic Press 1 (1965) 1-30.

[11] C.P. Slichter, Principles of Magnetic Resonance, Springer, Berlin; London, 1990.

[12] M. Goldman, J. Magn. Reson. 60 (1984) 437-452.

[13] A. Kumar, R.C.R. Grace, P.K. Madhu, Prog. NMR Spect. 37 (2000) 191-319.

[14] A. Kumar, P.K. Madhu, Conc. Magn. Reson. 8 (1996) 139-160.
[15] Mathematica, Version 5.1, Wolfram Research, Inc., Champaign, Illinois, 2004.

[16] A. Jerschow, J. Magn. Reson. 176 (2005) 7-14.

[17] S.A. Smith, T.O. Levante, B.H. Meier, R.R. Ernst, J. Magn. Reson. 106a (1994) 75-105.

[18] R.R. Ernst, G. Bodenhausen, A. Wokaun, Principles of Nuclear Magnetic Resonance in One and Two Dimensions, Clarendon Press, Oxford, 1987.

[19] J. Gendell, J.H. Freed, G.K. Fraenkel, J. Chem. Phys. 41 (1964) 949959.

[20] J.H. Freed, G.K. Fraenkel, J. Chem. Phys. 39 (1963) 326-348.

[21] B.C. Sanctuary, T.K. Halstead, Adv. Magn. Reson. 15 (1990) 79 161 .

[22] B.C. Sanctuary, F.P. Temme, Mol. Phys. 55 (1985) 1049-1062.

[23] B.C. Sanctuary, T.K. Halstead, P.A. Osment, Mol. Phys. 49 (1983) 753-784.

[24] B.C. Sanctuary, Mol. Phys. 48 (1983) 1155-1176.

[25] B.C. Sanctuary, J. Chem. Phys. 64 (1976) 4352-4361.

[26] D.M. Brink, G.R. Satchler, Angular Momentum, Clarendon Press, Oxford, 1993.

[27] D.A. Varshalovich, A.N. Moskalev, V.K. Khersonski, Quantum Theory of Angular Momentum: Irreducible Tensors, Spherical Harmonics, Vector Coupling Coefficients, 3nj Symbols, World Scientific, Singapore, 1988.

[28] G. Lipari, A. Szabo, J. Am. Chem. Soc. 104 (1982) 4546-4559.

[29] H. Desvaux, R. Kuemmerle, J. Kowalewski, C. Luchinat, I. Bertini, Chem. Phys. Chem. 5 (2004) 959-965.

[30] N. Wagner-Rundell, DPhil thesis, University of Oxford (2007).

[31] A.G. Palmer III, in: Protein NMR Spectroscopy, Principles and Practice, Academic Press, New York, 1996.

[32] R. Konrat, H. Sterk, Chem. Phys. Lett. 203 (1993) 75-80.

[33] M. Pellecchia, Y. Pang, L. Wang, A.V. Kurochkin, A. Kumar, E.R.P. Zuiderweg, J. Am. Chem. Soc. 121 (1999) 9165-9170.

[34] J. Czernek, R. Fiala, V. Sklenar, J. Magn. Reson. 145 (2000) 142-146.

[35] B.J. Wylie, W.T. Franks, C.M. Rienstra, J. Phys. Chem. B 110 (2006) 10926-10936.

[36] K.W. Waddell, E.Y. Chekmenev, R.J. Wittebort, J. Am. Chem. Soc. 127 (2005) 9030-9035.

[37] M.J. Frisch, G.W. Trucks, H.B. Schlegel, G.E. Scuseria, M.A. Robb, J.R. Cheeseman, J.A. Montgomery, T.V. Jr., K.N. Kudin, J.C. Burant, J.M. Millam, S.S. Iyengar, J. Tomasi, V. Barone, B. Mennucci, M. Cossi, G. Scalmani, N. Rega, G.A. Petersson, H. Nakatsuji, M. Hada, M. Ehara, K. Toyota, R. Fukuda, J. Hasegawa, M. Ishida, T. Nakajima, Y. Honda, O. Kitao, H. Nakai, M. Klene, X. Li, J.E. Knox, H.P. Hratchian, J.B. Cross, C. Adamo, J. Jaramillo, R. Gomperts, R.E. Stratmann, O. Yazyev, A.J. Austin, R. Cammi, C. Pomelli, J.W. Ochterski, P.Y. Ayala, K. Morokuma, G.A. Voth, P. Salvador, J.J. Dannenberg, V.G. Zakrzewski, S. Dapprich, A.D. Daniels, M.C. Strain, O. Farkas, D.K. Malick, A.D. Rabuck, K. Raghavachari, J.B. Foresman, J.V. Ortiz, Q. Cui, A.G. Baboul, S. Clifford, J. Cioslowski, B.B. Stefanov, G. Liu, A. Liashenko, P. Piskorz, I. Komaromi, R.L. Martin, D.J. Fox, T. Keith, M.A. AlLaham, C.Y. Peng, A. Nanayakkara, M. Challacombe, P.M.W. Gill, B. Johnson, W. Chen, M.W. Wong, C. Gonzale, J.A. Pople, Gaussian 03, Revision D.02, 2004.

[38] C. Knapp, N. Weiden, K.P. Dinse, Appl. Phys. A 66 (1998) 249255.

[39] C. Knapp, N. Weiden, H. Kass, K.P. Dinse, B. Pietzak, M. Waiblinger, A. Weidinger, Mol. Phys. 95 (1998) 999-1004.

[40] J.J.L. Morton, A.M. Tyryshkin, A. Ardavan, K. Porfyrakis, S.A. Lyon, G.A.D. Briggs, J. Chem. Phys. 124 (2006) 014508/1-014508/5. 